\title{
Nintedanib in the management of pulmonary fibrosis after COVID-19: a case report Chakrabortty $\mathbf{R}^{a}$, Rahman $\mathbf{S}^{b}$, Jahan $\mathbf{R}^{b}$, Dip $A H^{b}$, Rahman $M^{b}$
}

\begin{abstract}
Pulmonary fibrosis is becoming a recognized complication of coronavirus disease 2019 (COVID-19). Patients with pulmonary fibrosis may present with dry cough, shortness of breath, nail clubbing, low oxygen saturation. We report a case of a 40-year-old male patient with pulmonary fibrosis due to COVID19. Clinical examination showed that the patient was dyspneic with low oxygen saturation and there was bilateral inspiratory crepitation in the lower part of his chest. High resolution computed tomography showed bilateral multifocal patchy ground-glass opacities, consolidation with peripheral and basal distribution, sub-pleural fibrotic bands and vascular thickening (almost 40-45\% of parenchymal involvement). We prescribed him an antifibrotic drug, nintedanib and there was a significant clinical and radiological improvement after 15 days of treatment. Nintedanib may have novel therapeutic role in preventing COVID-19 associated fibrosis.
\end{abstract}

Key words: SARS-CoV-2, COVID-19, pulmonary fibrosis, antifibrotic drug, nintedanib.

(BIRDEM Med J 2021; 11(2): 148-152)

\section{INTRODUCTION}

In December 2019, a new virus named severe acute respiratory syndrome coronavirus 2 (SARS-CoV-2) emerged from Wuhan, China that causes respiratory problem. ${ }^{1}$ It spread worldwide rapidly and was declared a pandemic on 11th March 2020 by World Health Organization (WHO). As on March 6, 2021, approximately 11,68,86,214 confirmed cases and 2,59,404 deaths were declared throughout the world. Coronaviruses are an enveloped positive-sense RNA virus bind to angiotensin-converting enzyme 2 (ACE2) as their receptor. The virus must gain access to the host

\section{Author information}

a. Rajashish Chakrabortty, Associate Professor, Department of Respiratory Medicine, BSMMU, Dhaka, Bangladesh.

b. Samia Rahman, Rawnak Jahan, Abir Hasan Dip, Mohammed Mirazur Rahman, Phase-B Resident (MD-Pulmonology), BSMMU, Dhaka, Bangladesh.

Address of correspondence: Rajashish Chakrabortty, Associate Professor, Department of Respiratory Medicine, BSMMU, Dhaka, Bangladesh. Email: drrajashish@gmail.com

Received: April 10, 2021

Revision received: April 12, 2021

Accepted: April 13, 2021 cell cytosol after binding with the receptor. ${ }^{2}$ Symptoms of coronavirus disease 2019 (COVID-19) are diverse, ranging from mild upper respiratory tract symptoms to severe acute respiratory distress syndrome (ARDS). Pulmonary fibrosis is one of the sequel of COVID-19. Permanent pulmonary architectural distortion and irreversible lung dysfunction can occur due to pulmonary fibrosis. ${ }^{3}$ The major risk factors for severe COVID-19 are increasing age, male sex and comorbidities such as hypertension and diabetes and these severe infections are responsible for lung fibrosis. SARS-CoV2 attacks the tissue covering the lung alveoli causes profound fibrosis by deposition of excess collagen tissue in the lung. ${ }^{4}$ But the role of antifibrotic therapy in the treatment of patients with pulmonary fibrosis due to SARS-CoV-2 infection is poorly defined. ${ }^{5}$ Progressive, fibrotic, irreversible interstitial lung disease is defined by declining lung function, increasing the extent of fibrosis by computed tomography (CT), worsening symptoms and quality of life and early mortality. ${ }^{6,7}$ In this article, we describe the benefits of antifibrotic therapy in patients with ongoing SARS-CoV-2 virus 
infection to stop the worsening and aggravation of the clinical situation. It could have a role in preventing pulmonary fibrosis after SARS-CoV-2 infection and in accelerating the entire healing process.

\section{CASE REPORT}

A 40-year-old diabetic patient visited the Department of Respiratory Medicine, Bangabandhu Sheikh Mujib Medical University on 20th January 2021 with complaints of shortness of breath for 50 days. The non-smoker gentle man was diagnosed as a case of COVIDpneumonia on the basis of symptoms like fever, dry cough, shortness of breath and positive Reverse Transcription-Polymerase Chain Reaction for COVID19, on 4th December 2020. At that time his condition was rapidly deteriorating so that he needed intensive care unit (ICU) supports. In ICU he was treated with remdesivir, meropenem, moxifloxacin, steroid along with higher concentration of oxygen by high flow nasal cannula (HFNC). After 20 days, he was shifted from ICU to the cabin where he was treated with oxygen at 3-4 L/ minute to maintain oxygen saturation $94-95 \%$ along with other supportive treatment. He was discharged from the hospital on 4th January 2021. During discharge, his exertional dyspnea was persisting. It was gradually progressive in nature, so he was unable to perform his normal day-to-day activity and even shortness of breath persisted during rest. There is no previous history of any kind of heart or lung disease. So he consulted our respiratory department, BSMMU on an outdoor basis.

On general examination, the patient was looking ill with average nutrition and body built. He was dyspneic, respiratory rate 24 breaths/ $\mathrm{min}, \mathrm{SpO}_{2}$ was $92 \%$. Other vital signs within the normal limit. Respiratory system examination revealed bilateral fine inspiratory crepitation not altered after coughing. So, our diagnosis was COVID-19 associated pulmonary fibrosis.

On investigation, there was raised erythrocyte sedimentation rate (ESR). Previous CT scan (December 8,2020 ) revealed bilateral multifocal patchy ground-glass opacities with peripheral and basal distribution, consolidation, crazy paving, reverse halo sign, subpleural fibrotic bands and vascular thickening; findings suggested high suspicious of COVID-19. Total CT score was 12 out of 25 . Evidence of almost 45 to $50 \%$ of parenchymal involvement (Figure 1). Recent CT scan (January 17, 2021) revealed bilateral multifocal patchy ground-glass opacities with peripheral and basal distribution, consolidation, sub-pleural fibrotic bands and vascular thickening; findings suggest high suspicious of COVID-19. Total CT score 10 out of 25. Evidence of almost 40 to $45 \%$ of parenchymal involvement (Figure 2). Pulmonary function test revealed restrictive lung disease with markedly reduced DLCO. There was also reduced $6 \mathrm{MWD}$ with exercise induced oxygen desaturation.

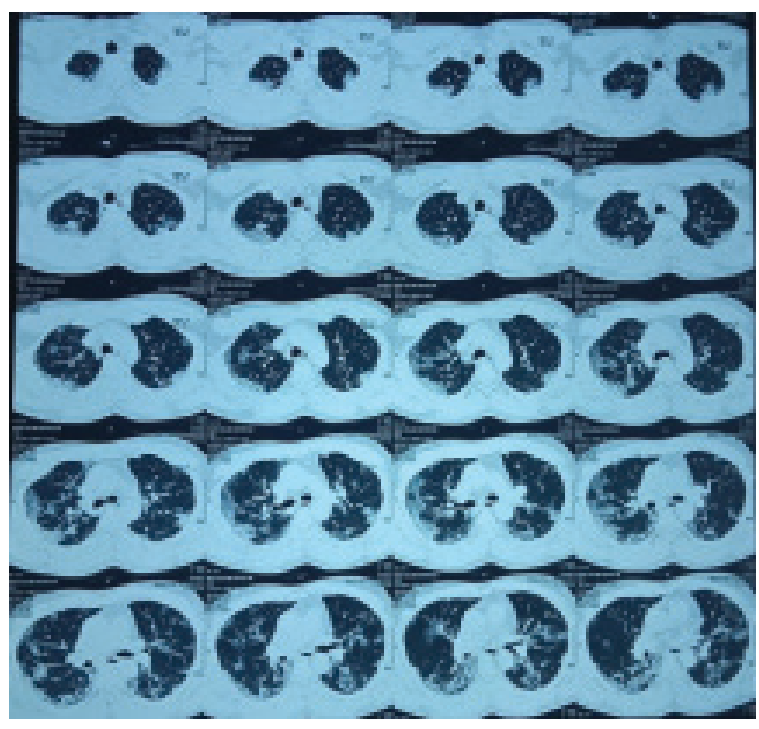

Figure 1 HRCT of the chest shows ground glass opacities with consolidation (at ICU)

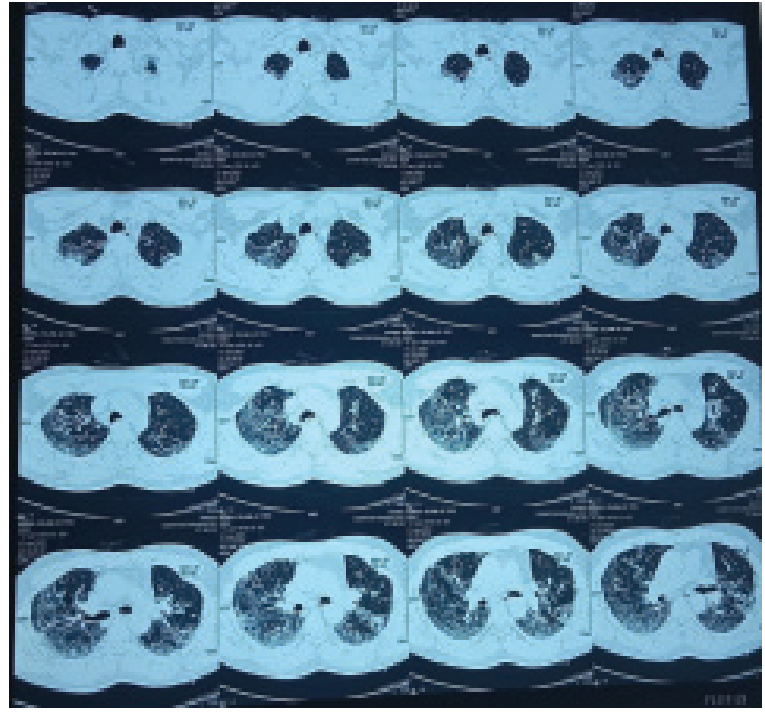

Figure 2 HRCT of the chest shows ground glass opacities with consolidation (after 50 days) 
As the patient was dyspneic and oxygen saturation $93 \%$ and there was no clinical or radiological improvement after 50 days of illness, the patient was prescribed antifibrotic drug, nintedanib, (150 mg) 12 hourly on 20th January 2021. After 15 days of treatment, the patient showed significant improvement both clinically and radiologically. His respiratory rate was 18 breaths/minute with an oxygen saturation of $98 \%$. CT scan (February 10, 2021) revealed multiple sub-pleural and parenchymal fibrotic band shadows were seen in both lungs with vascular thickening (significant improvement than previous) (Figure 3). Pulmonary function tests also showed marked improvement; FVC from $35 \%$ to $63.5 \%$, DLCO (corrected) from $3.8 \%$ to $45 \%$, total lung capacity from $5.4 \%$ to $61.7 \%$ and 6 -minute walk test showed 1305 feet walking distance with saturation of $95 \%$.

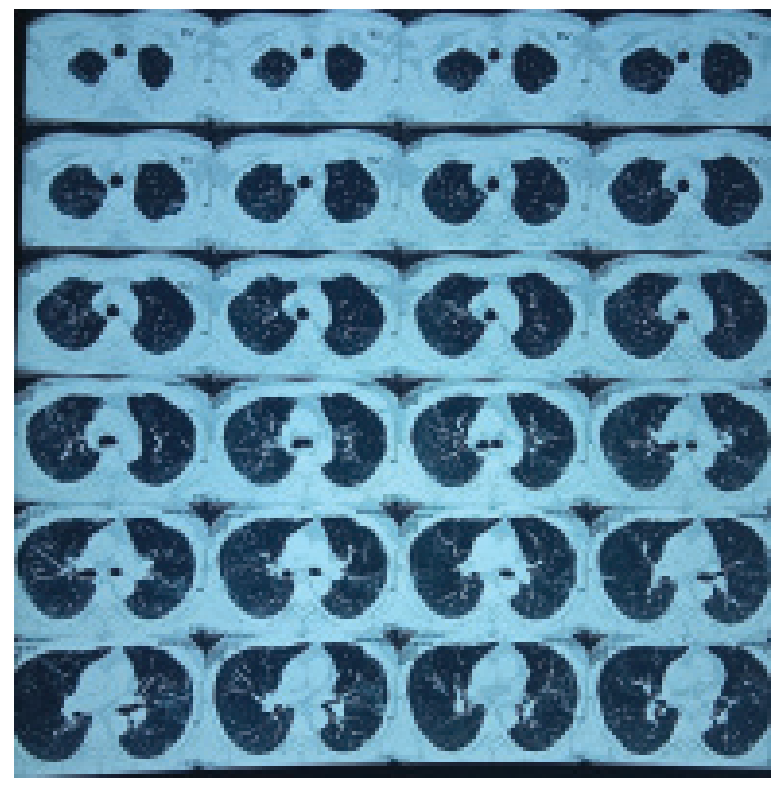

Figure 3 HRCT of the chest shows sub pleural and parenchymal fibrotic band shadow with vascular thickening (15 days of treatment with nintedanib)

\section{DISCUSSION}

While the long-term consequences of COVID-19 remain largely unidentified, there has been considerable assumption that patients with COVID-19 pneumonia, particularly severe or critical cases, are as more likely to develop pulmonary fibrosis as SARS and Middle East respiratory syndrome (MERS). ${ }^{9}$ Considering the rapidly evolving and increasing pandemic of COVID-19, the burden of pulmonary fibrosis after COVID-19 recovery will likely be considerable and become a major health apprehension worldwide. ${ }^{10}$ To date, there has been lack of therapeutic intervention either preventing or treating the pulmonary fibrosis as a consequences of COVID19.

One of the risk factors for the development of lung fibrosis in COVID-19 is advanced age. Second risk factor for increased disease severity is some comorbidity like hypertension, diabetes and coronary artery disease. Third and important risk factor is prolonged ICU stay and duration of mechanical ventilation. ${ }^{10}$ In our case , patient was diabetic and required ICU support for about 20 days.

As after binding with the ACE2 receptor, SARS-CoV-2 virus regulates renin-angiotensin system (RAS). The effects of increased angiotensin II include activation of interleukin (IL)-6, tumor necrosis factor-alpha (TNF alpha) and increased recruitment of neutrophils and macrophages as well as direct endothelial cell injury. ${ }^{13}$ Acute lung injury causes excessive production of reactive oxygen species (ROS) and release of TGF-b, FGF, VDGF and PDGF. Fibroblasts are recruited causes direct endothelial cell injury by deposition of collagen, extracellular matrix. As the wound-healing response is hampered, it results in reduced tissue function due to increased thickness, stiffening, altered vascularization and lastly pulmonary fibrosis. ${ }^{14}$

Patients affected with pulmonary fibrosis commonly complain of dry cough, fatigue and dyspnea. Weight loss is expected. Pulmonary fibrosis is a chronic, progressive and fatal lung disease that causes irreversible loss of lung function. These may lead to a decreased functional capacity, a decreased quality of life. ${ }^{15}$ If patient with pulmonary fibrosis remain untreated later may develop so many complications like pulmonary hypertension, respiratory failure.

Our patient presented with shortness of breath after COVID-19 infection, with low oxygen saturation and decreased exercise capacity. So, our diagnosis was pulmonary fibrosis. Like diffuse parenchymal lung disease [both idiopathic pulmonary fibrosis (IPF) and other than IPF], COVID-19 associated pulmonary fibrosis share the same pathogenesis. So, the antifibrotic agents that used to reduce or prevent fibrosis in idiopathic pulmonary fibrosis, maybe effective in COVID-19 associated fibrosis. Pirfenidone and 
nintedanib are antifibrotic drugs which have different mode of action, but effective to decline the rate of lung function. It can be a beneficial aid in the prevention of serious or fatal complications from COVID-19 or in those which already healed with residual fibrotic lung lesions. ${ }^{16,17}$

Nintedanib is an oral intracellular tyrosine kinase inhibitor. It inhibits downstream signaling pathways that involved in proliferation, migration and maturation of lung fibroblasts by inhibition of activation of plateletderived growth factor receptor, vascular endothelial growth factor receptor and fibroblast growth factor receptor. ${ }^{18,19}$

There are so many studies on nintedanib. The INPULSIS studies (INPULSIS 1, INPULSIS 2), in Phase III trials, to investigate the efficacy and safety of nintedanib 150 $\mathrm{mg}$ twice daily compared with placebo in patients with IPF. These studies showed that patients with IPF, nintedanib reduced the decline in $\mathrm{FVC}$, which is consistent with a slowing of disease progression. The commonest side effect on nintedanib is diarrhea. ${ }^{20} \mathrm{In}$ INBUILD trial for evaluating the efficacy and safety of nintedanib, over 52 weeks in patients with Progressive Fibrosing Interstitial Lung Disease (PF-ILD). The patients who received nintedanib had a slower progression of interstitial lung disease than those who received placebo, as shown by a lower annual rate of decline in the FVC over the 52-week period. ${ }^{21}$

Our patient showed significant improvement in FVC from $35 \%$ to $63.5 \%$. DLCO also improved. Patient's general condition also improved.

\section{Conclusion}

Nintedanib might provide a novel therapeutic approach for managing COVID-19-induced pulmonary fibrosis. Given the scale of the COVID-19 pandemic and the number of patients requiring invasive ventilation worldwide, post-COVID-19 pulmonary fibrosis is becoming a lethal threat to global health. Our patient was treated with nintedanib and got significant improvement in terms of radiological as well as laboratory parameters (Spirometry, DLCO). This drug could have therapeutic potential for treating severe COVID-19 and help to prevent pulmonary fibrosis. To deal with this emerging issue, trials evaluating the efficacy and safety of nintedanib against COVID-19related fibrotic complications of lung are necessary.
Authors' contribution: RC contributed in management of the patient and editing this article. SR, RJ and AHD drafted the manuscript. MMR edited the work. All authors read and approved the final manuscript.

Conflicts of interest: Nothing to declare.

Consent: Informed written consent was taken from patient regarding publication of this case report.

\section{REFERENCES}

1. Zhu N, Zhang D, Wang W, Li X, Yang B, Song J, et al. A novel coronavirus from patients with pneumonia in China, 2019. N Engl J Med 2020; 382:727-33

2. Fehr AR, Perlman S. Coronaviruses: an overview of their replication and pathogenesis. Methods Mol Biol 2015; 1282:1-23.

3. Al Mamun SA, Jahan R, Islam QT, Nazrin T, Shajalal K. Rationale of Using Common Antifibrotic Therapy in Post COVID Fibrosis. J Medicine 2021 Jan 14;22(1):46-50.

4. Chung MP, Park MS, Oh IJ, Lee HB, Kim YW, Park JS, et al. Safety and Efficacy of Pirfenidone in Advanced Idiopathic Pulmonary Fibrosis: A Nationwide PostMarketing Surveillance Study in Korean Patients. Adv Ther 2020;37(5):2303-16.

5. George PM, Wells AU, Jenkins RG. Pulmonary fibrosis and COVID-19: the potential role for antifibrotic therapy. Lancet Respir Med 2020; 8: 807-15.

6. Ghose M, Islam T. Facing the Challenge of Post COVID19 Pulmonary fibrosis: What is so unique about it? Bangladesh Critical Care Journal 2020 Nov 1;8(2):1027 .

7. Spagnolo P, Balestro E, Aliberti S, Cocconcelli E, Biondini D, Casa GD, et al. Pulmonary fibrosis secondary to COVID19: a call to arms? Lancet Respir Med 2020 Aug $1 ; 8(8): 750-2$.

8. McDonald LT. Healing after Covid-19: Are Survivors at Risk for Development of Pulmonary Fibrosis? Am J Phy Lung Cell Mol Phy 2021;320(1):257-65.

9. Vitiello A, Pelliccia C, Ferrara F. COVID-19 patients with pulmonary fibrotic tissue: Clinical pharmacological rational of antifibrotic therapy. SN Comprehensive Clinical Medicine 2020 Oct; 2(10):1709-12.

10. Rai DK, Sharma P, Kumar R. Post covid 19 pulmonary fibrosis- Is it reversible? [published online ahead of print, 2020 Nov 10]. Indian J Tuberc 2020;doi:10.1016/ j.ijtb.2020.11.003

11. Chaudhary S, Natt B, Bime C, Knox KS, Glassberg MK. Antifibrotics in COVID-19 Lung Disease: Let Us Stay Focused. Front Med 2020 Sep 9;7:539.

12. Ouassou H, Kharchoufa L, Bouhrim M, Daoudi NE, Imtara $\mathrm{H}$, Bencheikh N, et al. The Pathogenesis of coronavirus 
disease 2019 (COVID-19): Evaluation and prevention. J Immunol Res 2020 |1357983 | https://doi.org/10.1155/ 2020/1357983.

13. Wu Y, Guo C, Tang L, Hong Z, Zhou J, Dong X, et al. Prolonged presence of SARS-CoV-2 viral RNA in faecal samples. Lancet Gastroenterol Hepatol 2020 May $1 ; 5(5): 434-5$.

14. Gonzalez-Gonzalez FJ, Chandel NS, Jain M, Budinger GS. Reactive oxygen species as signaling molecules in the development of lung fibrosis. Translational Research 2017 Dec 1;190:61-8.

15. Alhiyari MA, Ata F, Alghizzawi MI, Bilal AB, Abdulhadi AS, Yousaf Z. Post COVID-19 fibrosis, an emerging complication of SARS-CoV-2 infection. IDCases 2021 Jan 1; 23:e01041.

16. King Jr TE, Bradford WZ, Castro-Bernardini S, Fagan EA, Glaspole I, Glassberg MK, et al. A phase 3 trial of pirfenidone in patients with idiopathic pulmonary fibrosis. N Engl J Med 2014 May 29;370(22):2083-92.
17. Guan WJ, Ni ZY, Hu Y, Liang WH, Ou CQ, He JX, et al. Clinical characteristics of coronavirus disease 2019 in China. N Engl J Med 2020 Apr 30;382(18):1708-20.

18. Collins BF, Raghu G. Antifibrotic therapy for fibrotic lung disease beyond idiopathic pulmonary fibrosis. Eur Respir Rev 2019 Sep 30;28:153.

19. Wollin L, Wex E, Pautsch A, Schnapp G, Hostettler KE, Stowasser S, et al. Mode of action of nintedanib in the treatment of idiopathic pulmonary fibrosis. Eur Respir J 2015 May 1; 45(5):1434-45.

20. Richeldi L, Du Bois RM, Raghu G, Azuma A, Brown KK, Costabel U, et al. Efficacy and safety of nintedanib in idiopathic pulmonary fibrosis. N Engl J Med 2014 May 29; 370(22):2071-82.

21. Flaherty KR, Wells AU, Cottin V, Devaraj A, Walsh SL, Inoue $\mathrm{Y}$, et al. Nintedanib in progressive fibrosing interstitial lung diseases. N Engl J Med 2019 Oct 31; 381(18):1718-27. 